ISSN: 1130-3743 - ISSN electrónico: 2386-5660

DOI: http://dx.doi.org/10.14201/teoredu201527291114

\title{
PENSAMIENTO Y ACCIÓN SOCIOEDUCATIVA EN CONTEXTOS DE ENSEÑANZA SECUNDARIA. UN ESTUDIO DESCRIPTIVO-CORRELACIONAL
}

\author{
Socio-educational thinking and action in school context. \\ A descriptive-correlational study
}

\section{Pensée et action socio-éducative dans des contextes de l'enseignement secondaire. Une étude descriptive et de corrélation}

Margarita GonzÁlez SÁnchez, Susana Olmos MigueláÑEz y Sara SERRATE GonzÁlez Universidad de Salamanca. Facultad de Educación. Departamento de Teoría e Historia de la Educación. Paseo de Canalejas, 169. 37008 Salamanca. mgsa@usal.es; solmos@usal.es; sarasg@usal.es

Fecha de recepción: marzo de 2015

Fecha de aceptación: junio de 2015

Biblid [(1130-3743) 27, 2-2015, 91-114]

\section{RESUMEN}

Este artículo refiere resultados de un proyecto de investigación más amplio que engloba diferentes unidades de análisis relacionadas con el educador social y su acción profesional. Pretendemos describir y justificar la intervención socioeducativa que desempeñan los educadores sociales en los centros de enseñanza, por este motivo planteamos en las comunidades de Castilla La Mancha y Extremadura un estudio descriptivo-correlacional, dentro del clásico diseño de encuesta, a través de un cuestionario. Los resultados corroboran la creciente irrupción de los educadores sociales en el sistema educativo y las funciones realizadas que nos permite revisar 
y debatir el papel de los educadores en los centros. Presentamos los resultados parciales que hacen referencia a una de las dimensiones estudiadas referidas, en líneas generales, al perfil del educador social y, más concretamente, a las funciones del mismo en los centros de secundaria colaborando en la respuesta que la sociedad actual demanda a la educación.

Palabras clave: Centros de enseñanza secundaria; intervención socioeducativa; funciones; acción profesional.

\section{SUMMARY}

This article provides findings from a broader research project that comprises different units of analysis related to social educators and their professional activity. Here we describe and justify social-educational interventions by social educators in schools. To this end a descriptive-correlational study using a classic survey design with questionnaire was carried out in Spain's autonomous regions of Castile-La Mancha and Extremadura. Our findings corroborate the growing presence of social educators in the educational system and the functions they perform, allowing us to review and discuss the role of social educators in schools. Here we present partial results addressing one of the dimensions studied, referring in general to the professional profile of social educators and more specifically to their functions in schools of secondary education as they respond to the demands that today's society expects from the educational system.

Key words: Secondary schools; social-educational intervention; functions; professional activity.

\section{SOMMAIRE}

Cet article présente les résultats d'un projet plus étendu de recherche qui comporte différentes unités d'analyse à propos de l'éducateur spécialisé et son action professionnelle. Pour décrire et justifier l'intervention socio-éducative menée par les éducateurs spécialisés dans les centres d'enseignement, une étude descriptivecorrélationnelle a été réalisée dans les Communautés Autonomes de Castilla la Mancha et de Extremadura au moyen d'un questionnaire crée dans le cadre classique des enquêtes. Les résultats corroborent l'irruption croissante des éducateurs spécialisés dans le système éducatif et les fonctions qui leur sont attachées, ce qui permet de réviser et de débattre le rôle des éducateurs dans les centres. On y présente des résultats partiels d'une des dimensions étudiées concernant, grosso modo, le profil de l'enseignant spécialisé et, plus en particulier, les fonctions qui lui sont attachées dans les centres d'enseignement secondaire pour répondre aux demandes que la société actuelle pose à l'éducation.

Mots clés: Centres d'enseignement secondaire; intervention socio-éducative; fonctions; action professionnelle. 
M. GONZÁLEZ SÁNCHEZ, S. OLMOS MIGUELÁÑEZ Y S. SERRATE GONZÁLEZ

\section{INTRODUCCIÓN}

Cada época ha exigido de la educación respuestas acordes a los problemas a los que la sociedad se enfrentaba en cada momento. La sociedad actual del conocimiento y de la información, la sociedad red, se encuentra en un momento de adaptación frente a los avances tecnológicos, movimientos migratorios, globalización de mercados, que producen cambios notables que afectan a la organización social reflejada en nuestros hábitos de vida donde cada vez están más presentes la pluralidad y la interculturalidad.

Entendiendo la educación como prioridad estratégica para la mejora de nuestra sociedad, consideramos que las instituciones de educación deberán adaptarse a las necesidades y a los cambios que convergen en nuestra sociedad cada vez más compleja, global y dinámica. Una educación que como proceso de desarrollo personal y social debe tener como referente el contexto que la envuelve, donde se encuentra con nuevas demandas, situaciones y algunos desafíos de la realidad compleja actual que provocan que deba ser repensada, comenzando por redefinir conceptual y funcionalmente su ser y quehacer. Se hace necesario pues plantearnos qué funciones, además de transmitir información, de instruir, se requiere hoy desarrollar para que siga siendo eficaz y eficiente a las necesidades y demandas sociales (Bolívar, 2013). Esta realidad nos hace pensar en la incorporación de personal cualificado como el profesional de lo social para asumir y/o colaborar en las nuevas preocupaciones de desarrollo formativo y social.

En la necesidad de abordar la realidad desde una perspectiva globalizadora, teniendo en cuenta los distintos factores que interactúan en el proceso educativo en el mismo sistema escolar, como en la relación de este con otros sistemas e instituciones, encontramos, tiene su razón de ser y se avala la pertinencia de profesionales de la educación social.

Nos encontramos ante un sistema con entidad propia, conformado por diversos subsistemas (alumnos, profesores, equipo directivo, profesionales especializados) a los que ha de hacer convivir, estableciendo secuencias de interacción y reglas específicas de comunicación. La configuración de este sistema implica la construcción de un modelo de convivencia para afrontar la variedad de situaciones y reflejos del exterior, problemas o situaciones a los que deben enfrentarse los centros escolares y que deben ser analizados con referencia a su estructura interna y tener como referencia el entorno que les rodea. De este modo, los centros educativos no pueden ofrecer solución a determinadas situaciones de necesidad aislada sin tener en cuenta el comportamiento del resto de elementos que interactúan simultáneamente (Krenz, Cassas, Aguilar y Carbó, 2009).

Dar respuestas a las demandas de la sociedad y ofrecer alternativas socioeducativas a la situación contextual del momento implica recurrir a la perspectiva holística, integradora y sistémica del sistema escolar (Bertalanffy, 1976) como marco interpretativo en el que poder entender y situar las relaciones y conjuntos que emergen entre sociedad y escuela y configurar, de manera interdisciplinar, un 
cuerpo profesional que desarrolle un trabajo conjunto, programado y coordinado que redunde en la educación y socialización de la comunidad educativa.

Sin duda, es importante y respetable el papel que desempeñan los centros escolares en la sociedad como agente educativo, pero es una evidencia que existen dificultades que afectan, en distinta medida, a la vida cotidiana en los centros, como: la violencia en las aulas y el incremento del conflicto (Córdoba, Del Rey y Ortega, 2010; Mazorra, 2008), el elevado índice de fracaso escolar, el aumento de alumnado inmigrante (Fernández, Mena y Riviere, 2010; Rahona y Morales, 2013) o el impacto que tienen las nuevas tecnologías en la configuración de las relaciones sociales (Pérez de Guzmán, 2008). Todo ello promueve que los centros tengan que transformarse en algo diferente si quieren dar solución a dichas problemáticas, en consecuencia, deberán redefinir conceptual y funcionalmente "su ser y quehacer para dar respuestas acordes a las necesidades de la sociedad actual» (Pérez, 2008, 8).

La respuesta a este escenario requiere intervenciones educativas de profesionales complementarios a los docentes, que suponen una oportunidad para aportar cambios que podemos encontrar en el campo de la educación social, en tanto que pueden colaborar y actuar conjuntamente con el profesorado en el espacio de los centros a la solución de las dificultades que se le presentan desde la sociedad actual como competencia de la escuela. Pensamos que este es el espacio del profesional de la educación social, desde donde se propone un tipo de educación, como afirma Ortega (2005), diferenciada en parte de la educación escolar, que busca la activación de las condiciones educativas de la cultura y de la vida social, pero también la prevención, compensación o reeducación de la dificultad y el conflicto social, dentro del marco general que configura a la finalidad integradora de toda la educación.

Entre los problemas propios del contexto que influyen en la actividad cotidiana de los centros de enseñanza, más concretamente en los centros de secundaria, se encuentran el fenómeno de la interculturalidad, los problemas de violencia y acoso escolar, el absentismo y abandono temprano unidos al fracaso escolar, la nueva configuración y funciones familiares, los nuevos retos didácticos de las tecnologías en el aula y sus implicaciones en el desarrollo, así como la necesidad de atender las actuales alfabetizaciones, la configuración de las relaciones sociales de las generaciones más jóvenes, la convivencia pacífica dentro del propio centro entre los miembros de la comunidad educativa, como la relación de los centros educativos con las instituciones sociales próximas al mismo como ponen de manifiesto, entre otros, autores como Beltrán, 2007; Bernete, 2010; De León, 2011; Estrada, 2012; Gobernado, 2007; González y Serrate, 2013; Martínez y De Andrés, 2011; Matamales, 2007; Moreno y Suárez, 2010. En un intento de dar respuesta a estas situaciones que afectan al sistema escolar, existe un amplio abanico de líneas de investigación tanto a nivel nacional como internacional. Algunas de estas investigaciones más recientes y relevantes se encaminan a examinar los diversos mecanismos existentes de gestionar la convivencia escolar (Conde, 2012), estudiar el fenómeno de la violencia y su evolución más actual conocida como cyberbullying, 
M. GONZÁLEZ SÁNCHEZ, S. OLMOS MIGUELÁÑEZ Y S. SERRATE GONZÁLEZ

las vías a través de las cuales se produce, su prevalencia en la etapa de secundaria y las consecuencias en el rendimiento escolar y social (Garaigordobil, 2011; MoraMerchán, Ortega, Calmaestra y Smith, 2010; Ortega, Calmaestra y Mora-Merchán, 2008; Ortega-Reyes y González-Bañales, 2015). En otro orden de cosas encontramos estudios orientados a la búsqueda de propuestas para fomentar una adecuada integración del alumnado extranjero en el sistema educativo, tanto español como en el de otros países europeos (Durk, 2012), analizar la relación de las familias con la escuela en la etapa de secundaria (Moreno, 2010), los determinantes del riesgo de fracaso escolar, así como los factores que producen abandono temprano (Calero, Choi y Waisgrais, 2010; CSFT, 2013). En torno al objetivo principal de este artículo, se han realizado diversos estudios en los que se evalúan el perfil y las funciones profesionales del educador social (Losada, Muñoz y Espiñeira, 2015; Vallés, 2011), se examinan las competencias que deben caracterizar a los educadores sociales, tanto en este como en otros campos, para intervenir socioeducativamente con adolescentes y jóvenes (Bautista-Cerro y Melendro, 2011) y se analiza el papel del educador social en la etapa de secundaria (Barranco, Díaz y Fernández, 2012; Ortega et al., 2010; Sarrado y Fernández-Díaz, 2012).

Desde un planteamiento global de la educación, integradora de los diferentes procesos formativos que confluyen en nuestra sociedad, estamos convencidos de que se deben articular mecanismos diversificados para afrontar estas situaciones y desafíos sociales que requieren de otros conocimientos y profesionales que apoyen y complementen a los centros educativos en su intervención.

En la línea de las investigaciones y estudios existentes, abordamos la concreción de un referente profesional en los centros escolares como es el educador social y sus funciones, constitutivas de su perfil profesional a partir de la investigación desarrollada ${ }^{1}$, donde se enmarcan parte de los resultados que aquí presentamos.

\subsection{Los profesionales de intervención socioeducativa en la etapa de secundaria}

La actividad educativa no puede quedar al margen de los problemas de su entorno. Para hacer frente a los actuales retos educativos se requieren profesionales con una consistente formación disciplinar, amplia y diversificada, como una formación profesional con capacidad de adaptación a las nuevas situaciones sociales implicando en el proceso educativo a cuantas instancias resulte necesario. Tal y como apunta Tribó (2008), el perfil de los profesionales del siglo xxi que actúan en educación secundaria comporta un cambio epistemológico o de concepto, en tanto es necesario situar los procesos de aprendizaje de los alumnos como ejes centrales y acentuar la capacidad de construir conocimiento en actuaciones interdisciplinares para fomentar la integración y participación del

1. Proyecto de investigación: Situación, praxis y demandas profesionales de intervención socioeducativa en el sistema escolar de Castilla y León. Referencia: SA05508. Entidad: Junta de Castilla y León. 
M. GONZÁLEZ SÁNCHEZ, S. OLMOS MIGUELÁÑEZ Y S. SERRATE GONZÁLEZ

PENSAMIENTO Y ACCIÓN SOCIOEDUCATIVA EN CONTEXTOS DE ENSEÑANZA SECUNDARIA...

alumnado en la sociedad, superando los desafíos sociales a los que tendrán que enfrentarse y persiguiendo "capacitar al alumno para que formule y sea capaz de llevar libremente a la práctica su proyecto personal de vida, es decir, formar la persona autónoma» (Pérez, 2010, 208).

Si bien es cierto que los currículos actuales se encuentran sobrecargados de disciplinas y materias encaminadas a la formación del alumnado (Pineda y García, 2014), se deben priorizar aquellos conocimientos que tienen un carácter emancipatorio, que permiten al alumnado madurar y convertirse en personas adultas para enfrentarse (integrarse competentemente) a la sociedad. En este sentido, una coherente y eficaz intervención, tanto por parte del profesorado como por parte de profesionales especializados de apoyo, implica tener en cuenta el perfil psicopedagógico del alumnado de secundaria en cuanto a edad, contexto socioeducativo en el que se ubican, posibilidades de aprendizaje, etc. (Unceta, 2006), para tomar decisiones correctas ante las nuevas situaciones que se presentan. Esta toma de decisiones no debe recaer únicamente en el profesorado que se encuentra en las aulas, y que debe saber trabajar en equipo a través de proyectos interdisciplinares, una de las características de la cultura docente eficiente (Tribó, 2008). Este saber trabajar en equipo implica estar coordinado con profesores de otras áreas en secuencias instructivas y con profesionales que colaboran con los docentes y forman parte del proceso de enseñanza-aprendizaje de los alumnos, y en la resolución de problemáticas concretas, actuando como figuras mediadoras en el contexto escolar, y entre este y las familias o el contexto social cercano con miras a la formación personal, social y ciudadana.

Es evidente que los cambios en el contexto macrosocial refieren otras exigencias y plantean al sistema escolar un importante desafío, el sistema no puede responder promoviendo solo aspectos cognitivos, olvidando que es un espacio de socialización privilegiado y debe responder al desarrollo de otras dimensiones del desarrollo humano en una dimensión más vital, que hace necesario desarrollar nuevas formas de actuación que es evidente que requieren una intervención integral, desde una perspectiva de trabajo en equipo, lo que exige plantearse acciones dirigidas a los alumnos, a las familias y a las diferentes instituciones y agentes sociales a la vez que se les hace partícipes en la responsabilidad de la educación. Estamos pensando en respuestas a situaciones de conflicto que se dan en las relaciones, promoción de la convivencia, la atención a la diversidad, la falta de disciplina, educación en valores, prevención y resolución de conflictos sociales, compensación de desigualdades, etc.

La finalidad de contar con nuevos y diversos profesionales en los centros de secundaria es incrementar la calidad de la educación y ello implica prestar atención a la mejora, cualitativa y cuantitativa, de los centros, concentrando los esfuerzos en que desarrollen sus capacidades internas para el cambio (Martínez, Krichesky y García, 2011), fomentando las fortalezas del centro y de sus miembros para conseguir solventar las dificultades que en ellos se presenten o generen. Como una de las respuestas sociales a esta demanda de incorporación de nuevas 
M. GONZÁLEZ SÁNCHEZ, S. OLMOS MIGUELÁÑEZ Y S. SERRATE GONZÁLEZ

PENSAMIENTO Y ACCIÓN SOCIOEDUCATIVA EN CONTEXTOS DE ENSEÑANZA SECUNDARIA...

figuras profesionales de la intervención socioeducativa, entre ellos, los educadores sociales.

En este sentido, determinadas Comunidades Autónomas, como es el caso de Extremadura, Castilla La Mancha ${ }^{2}$ o Andalucía cuentan con educadores sociales entre las plantillas de profesionales que intervienen en la etapa de secundaria, que suponen una nueva herramienta disciplinar en esta idea de mejorar los centros de enseñanza a través de propuestas en las que se potencia la capacidad interna de los mismos (Hargreaves y Fink, 2006).

La llegada de los educadores sociales a los centros de secundaria implica un cambio estructural dentro del sistema escolar (Sánchez, 2008), contemplado como un profesional que ofrece oportunidad de cambio y opciones renovadas para que el sistema educativo pueda hacer frente a la resolución de sus problemas, ofreciendo nuevas metodologías y dinámicas de trabajo, integrando una pedagogía de naturaleza más social en los centros. Esto es lo que Castillo denomina "paso de una óptica curricular a una perspectiva socioeducativa en la escuela" (Castillo, 2008, 65; 2013), que lleva asociadas unas connotaciones pedagógicas y un cambio en los objetivos y en la finalidad de la educación escolar.

En base a lo anterior, el educador social constituye una figura que acompaña de forma responsable e integral a los alumnos ayudándoles a convertirse en ciudadanos participativos, responsables y críticos con el entorno que les rodea (Tribó, 2008), ofreciendo herramientas y mecanismos en aquellos aspectos que repercutan en su bienestar e influyan en su desarrollo.

Su preparación, a nivel pedagógico, comunitario y mediador, le ofrece la posibilidad de realizar su trabajo en los centros de secundaria con todos los miembros de la comunidad educativa, integrado dentro de los departamentos y equipos de orientación. Se convierte así en un agente de cambio interno, a través de actuaciones sistémicas cuyos objetivos máximos son optimizar el logro académico de los estudiantes, establecer relaciones de trabajo colaborativo con el equipo de docentes (Martínez, Krichesky y García, 2011) y establecer conexiones duraderas y cercanas con los profesionales del entorno cercano.

La figura del educador social materializada en los centros toma forma, puesto que se trata de un profesional que por sus competencias y formación específica está capacitado para colaborar con intervenciones socioeducativas concretas y específicas (Barranco et al., 2012), junto al resto de profesionales, desarrollando funciones de orden preventivo, resolución de problemas y a través de actuaciones

2. La actuación de los educadores sociales en el espacio escolar viene avalada por normativa autonómica que regula la inclusión y funciones de este profesional. En Extremadura el V Convenio Colectivo para el personal laboral al servicio de la Junta de Extremadura. Resolución de 13 de julio de 2005 ( DOE 85, de 23 de julio) y Resolución de 16 de septiembre de 2005 (DOE 111, de 24 de septiembre). En Castilla La Mancha, Orden de 26 de junio de 2002 de la Consejería de Educación y Cultura por la que se desarrollan medidas contempladas en el Plan para la Mejora de la Eso. En Andalucía, Resolución de 16 de octubre, de la Dirección General de Gestión de Recursos Humanos, se convoca la cobertura provisional en 2006-2007 de puestos de educación social en el ámbito educativo. 
mediadoras de conflictos escolares, familiares y sociales, como también tareas de acompañamiento en situaciones de riesgo.

No obstante, uno de los aspectos que más preocupan al resto de profesionales que intervienen en los centros de secundaria son las tareas que estos profesionales tienen asignadas. Por ello, no recurrimos a un debate nuevo cuando tratamos de acotar las funciones profesionales del educador social en el entorno educativo, sus espacios de intervención y la forma en la que deben coordinarse con el resto de agentes (Guerrero y Rubio, 2008), pues desde hace tiempo se viene demandando la superación en la ambigüedad de las tareas atribuidas al educador social con el objetivo de «dotar de mayor relevancia y participación a esta figura de vital importancia en la institución educativa» (Sánchez, 2008).

Consideramos que las funciones de los educadores sociales deben ser planteadas según la práctica cotidiana de su trabajo en los centros de secundaria, desde donde se traducen las funciones en acciones más concretas, ejercidas de acuerdo con los problemas y necesidades que se atienden (Sáez, 2005). Por lo tanto, creemos relevante, en este contexto, verificar las funciones que los educadores acometen en su actuación profesional en el sistema educativo en aras de responder, al menos, a parte de las demandas que plantea la realidad social a la institución escolar.

Es por ello que, buscando precisar la emergente respuesta de la educación social escolar, el objetivo del estudio que presentamos es identificar las funciones y actividades que realizan los educadores sociales en los centros de secundaria de Extremadura y Castilla La Mancha. Centramos la investigación en el propio escenario de actuación profesional, concretamente en estas dos Comunidades Autónomas pioneras en incorporar educadores sociales en centros de secundaria, próximas a nuestro entorno sociogeográfico.

\section{Metodología de inVESTigación}

\subsection{Objetivo del estudio}

El estudio que presentamos, enmarcado en el campo de la educación social y de la investigación socioeducativa, pretende identificar las funciones y actividades que realizan los educadores sociales en el Sistema Escolar de Extremadura y Castilla La Mancha.

El diseño del estudio ha sido de tipo descriptivo-correlacional, englobándose dentro de las metodologías ex-post facto (Arnal, Del Rincón y Latorre, 1992). Se plantea un estudio de encuesta (Kerlinger y Lee, 2002) que nos permite conocer, a través de preguntas, las actividades y funciones que realizan los educadores sociales en los centros escolares en las comunidades estudiadas. Se observa, de entrada, una diferencia inicial entre ambas comunidades. En la primera, la normativa regula y establece un educador social por centro educativo, mientras que en la segunda 
M. GONZÁLEZ SÁNCHEZ, S. OLMOS MIGUELÁÑEZ Y S. SERRATE GONZÁLEZ

PENSAMIENTO Y ACCIÓN SOCIOEDUCATIVA EN CONTEXTOS DE ENSEÑANZA SECUNDARIA...

todavía no se contempla normativamente este profesional en todos los centros de enseñanza, dotándose, en su caso, de un educador si se argumenta su necesidad.

\subsection{Participantes}

Se invitó a participar a los 250 educadores en activo en los centros educativos de Extremadura y a los 49 educadores que trabajan en diferentes centros de Castilla La Mancha. La muestra final del estudio quedó conformada en un total de 108 educadores, 81 pertenecientes a Extremadura y 27 profesionales de Castilla La Mancha.

\subsection{Variables e instrumento de recogida de información}

Como variable compleja del estudio se consideraron "las funciones del educador social en los centros escolares"; y como variables de clasificación las definidas en la configuración de la muestra con respecto al centro (ubicación del centro, rural/urbano, tipo de centro, público/privado, etc.) y aquellas vinculadas directamente con el perfil del profesional que responde a la encuesta (titulación de acceso, años en el puesto de trabajo, género, lugar habitual de su puesto de trabajo, etc.).

El instrumento de recogida de datos es de naturaleza cuantitativa, en su mayor parte, basado en la metodología de encuesta estructurada y autoadministrada a través del envío postal, con preguntas cerradas, de alternativas tanto excluyentes como no excluyentes, con escalas dicotómicas en algunos casos, y valorativas en otros, así como una serie de ítems en escala tipo Likert (Morales, Urosa y Blanco, 2003) junto a ítems de respuesta abierta. El cuestionario consta de dos bloques como se puede apreciar en la Tabla 1:

TABla 1. Categorías del Cuestionario utilizado

\begin{tabular}{|l|ll|}
\hline $\begin{array}{l}\text { PERFIL PROFESIONAL } \\
\text { (Cuatro ítems) }\end{array}$ & - Titulación de acceso \\
& - & Sexo de nacimiento \\
\hline $\begin{array}{l}\text { VALORACIÓN DE LA ACTIVIDAD } \\
\text { PROFESIONAL (Dos ítems) }\end{array}$ & - & $\begin{array}{l}\text { Frecuencia en la realización de las funciones } \\
\text { Importancia de la funciones desempeñadas }\end{array}$ \\
\hline
\end{tabular}

En cuanto a validez de contenido, se ha garantizado a través de la valoración por jueces expertos sobre los enunciados de las preguntas planteadas. Para ello el primer borrador fue valorado, a partir de criterios de claridad y pertinencia, por un equipo formado por expertos académicos en educación social (4), metodología 
de investigación en educación (2) y profesionales de la educación social (2). Se efectuaron las modificaciones oportunas y se elaboró la encuesta definitiva.

Se intentó paliar el efecto de los errores de muestreo mediante la aplicación del cuestionario a toda la población, aumentando por tanto el tamaño de muestra recogida. Por otro lado, se redujeron los errores de medida al estandarizar las condiciones de aplicación, mediante instrucciones que garantizaran la igualdad de condiciones para todos los encuestados.

Aplicamos el coeficiente de fiabilidad de Alpha de Cronbach por ser uno de los más utilizados en el método de consistencia interna. El resultado obtenido en cada uno de los casos es el siguiente: 0,72, aunque el valor mínimo aceptable para dicho coeficiente es 0,70 , siendo conscientes que los valores designados deben preferiblemente oscilar entre 0,80 y 0,90 (Morales et al., 2003).

\subsection{Análisis de datos}

Para el análisis estadístico de los datos se han utilizado técnicas descriptivas (de tendencia central y de desviación) y correlacionales (Prueba T), utilizando para ello el paquete estadístico spss v.21.

\section{Resultados del estudio}

\subsection{Características de los encuestados}

Se destaca, en primer lugar, que, en la distribución en función del sexo de los participantes, el porcentaje de hombres fue inferior en ambas comunidades respecto al de mujeres. Así, en Castilla La Mancha un 85,2\% son mujeres y un 14,8\% hombres; y en Extremadura el $79,7 \%$ son mujeres y el $20,3 \%$ restantes hombres.

En relación a la titulación de acceso al puesto de trabajo, en ambas comunidades, son mayoritariamente titulados en Educación Social. En Castilla La Mancha un $59,3 \%$ son diplomados en dicha titulación frente a un $40,7 \%$ en otras titulaciones afines (14,8\% en Pedagogía, 7,4\% Psicopedagogía y el 18,5\% restante engloba otras titulaciones). En Extremadura, se observa un porcentaje más elevado, 78,8\% de acceso a través de Educación Social.

Los mayores porcentajes respecto a la edad de los participantes los encontramos, en Extremadura, en los 30 y los 34 años, abarcando un 9,2\% cada una, seguidos de los participantes con 33 años, con un 7,9\%. En Castilla La Mancha los mayores porcentajes los encontramos en los 30 y 31 años abarcando cada edad un 15,4\%, seguidos de aquellos participantes que tienen 51 años, un 11,5\% de la muestra. Estos datos nos indican que los puestos de educador social están siendo ocupados mayormente por jóvenes titulados que acceden a estas plazas de reciente creación. No obstante, hay un conjunto de población que accede a estos puestos por sistemas de promoción interna, son los casos de los participantes de mayor 
edad que, además, pueden o no poseer la titulación en Educación Social como se observa, principalmente, en la Comunidad de Castilla La Mancha.

Respecto al ámbito socioeducativo profesional en el que trabajan los educadores sociales encuestados, se observa mayoritariamente, como ámbito principal, los Departamentos de Orientación; así lo muestra el 81,5\% de las respuestas en Castilla La Mancha y el 98,8\% en Extremadura; tan sólo el 18,5\% trabajan en ámbitos distintos en Castilla La Mancha y en Extremadura el 1,2\% en equipos multiprofesionales.

\subsection{Valoración de las funciones que desempeñan los educadores, de acuerdo a la frecuencia y a la importancia}

Para realizar un análisis concreto del trabajo que desempeñan los educadores sociales en ambas Comunidades Autónomas, se solicitó a los encuestados que indicasen, para cada una de las funciones propuestas, la frecuencia de ejecución y la importancia que le otorgaban para un adecuado ejercicio profesional, teniendo en cuenta la escala siguiente: $1=$ nada, $2=$ poco, $3=$ bastante $y 4=$ mucho.

TABla 2. FunCiONES A DESARROLLAR POR LOS EDUCADORES SOCIALES EN LOS CENTROS ESCOLARES

\begin{tabular}{|r|l|}
\hline \multicolumn{2}{|c|}{ FunCIONES } \\
\hline 1. & Detección y prevención de factores de riesgo \\
\hline 2. & Mediación en conflictos de convivencia y su resolución \\
\hline 3. & Organización y gestión de servicios culturales, de ocio, etc. \\
\hline 4. & Diseño y desarrollo de programas de intervención intercultural \\
\hline 5. & Diseño y desarrollo de programas de acogida \\
\hline 6. & Diseño y desarrollo de programas de atención a la diversidad \\
\hline 7. & Diseño y desarrollo de programas de cooperación educativa \\
\hline 8. & Diseño y desarrollo de programas de comunicación interpersonal \\
\hline 9. & Desarrollo de programas de animación y participación \\
\hline 10. & Organización y desarrollo de escuela de padres, etc. \\
\hline 11. & Desarrollo de programas de orientación \\
\hline 12. & Desarrollo de programas contra absentismo escolar \\
\hline 13. & Organización de actividades extraescolares \\
\hline 14. & Participar en el desarrollo de habilidades sociales \\
\hline 15. & Actividad docente \\
\hline 16. & Función tutorial en un grupo docente \\
\hline 17. & Función tutorial profesional, prácticas \\
\hline
\end{tabular}




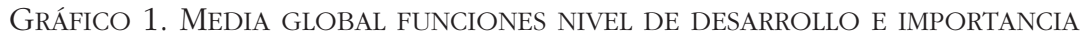

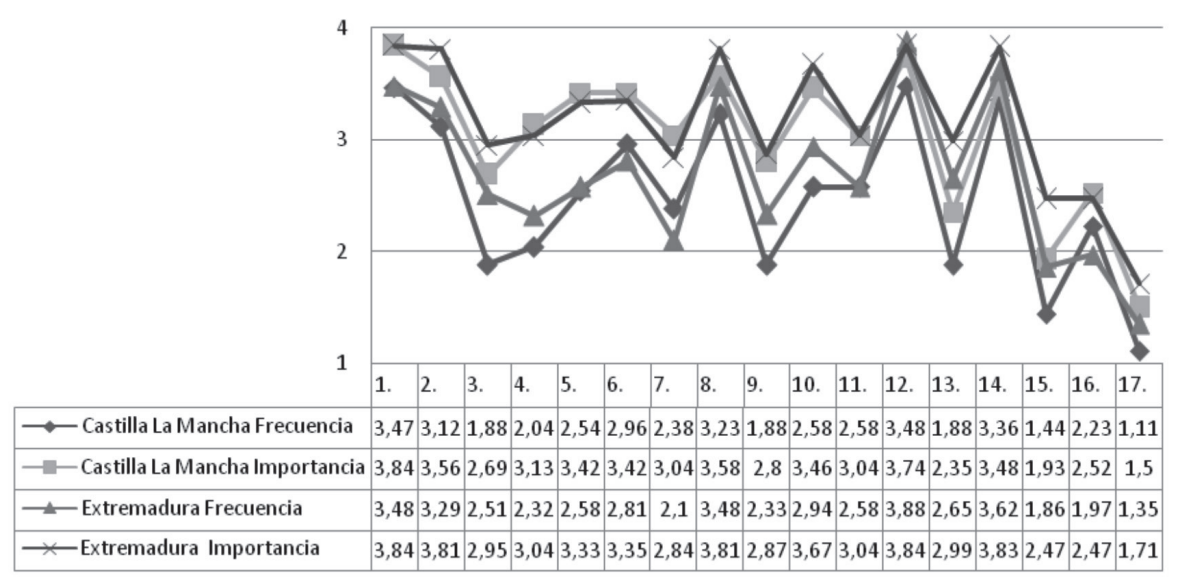

En ambas comunidades se observa que las funciones que los educadores sociales desempeñan en los centros se corresponden con las funciones dirigidas a la detección de factores de riesgo y mediación en conflictos de convivencia, así como aquellas dirigidas al diseño de programas contra el absentismo escolar, el fomento de la comunicación interpersonal y el desarrollo de habilidades de sociales. De igual forma, estas funciones son valoradas como las más importantes, haciendo hincapié también en la organización y desarrollo de escuelas de padres (Castilla La Mancha) y el desarrollo de programas de animación y participación (Extremadura) como tareas importantes a desarrollar por el educador social.

Las funciones menos desarrolladas en ambas Comunidades Autónomas son aquellas que corresponden a los ítems 15, 16 y 17, que podemos considerar funciones que no se corresponden con el perfil propiamente socioeducativo del educador social, perteneciendo más a tareas del profesorado. Llama la atención que entre las funciones que menos realizan los educadores se encuentren también la organización y gestión de servicios culturales y el desarrollo de programas de animación y participación, puesto que los propios educadores reconocen ser tareas importantes en su ejercicio cotidiano y representan funciones que definen un aspecto esencial del educador social en los centros, como es la promoción cultural y el fomento de la relación del centro escolar con el entorno (González y Serrate, 2013). Es posible considerar que, por falta de tiempo o de recursos, los educadores sociales no puedan abarcar el elenco de funciones que tienen asignadas y que, por ello, deban priorizar determinados aspectos en su ejercicio diario, relegando así otras funciones importantes a otros profesionales o a otros/as momentos/estancias.

Los encuestados, a partir de los ítems de respuesta abierta, nos ofrecieron un abanico amplio de tareas que desarrollan habitualmente, no incluidas en el 
M. GONZÁLEZ SÁNCHEZ, S. OLMOS MIGUELÁÑEZ Y S. SERRATE GONZÁLEZ

cuestionario, de donde extraemos que las funciones más desarrolladas y/o a las que confieren mayor relevancia en Castilla La Mancha se relacionan con la gestión y el trabajo en las aulas de convivencia y con el asesoramiento al equipo directivo, al profesorado y a las familias. En este contexto comunitario, el educador social es consciente de que representa un recurso interno de asesoramiento ${ }^{3}$ y apoyo tanto para la dirección de los centros como para el resto de agentes educativos, en dos direcciones concretas como son la promoción y mejora de la convivencia escolar y la prevención y el seguimiento del absentismo, de ahí sus respuestas.

Mientras que en Extremadura se resaltaron funciones como el préstamo de material e información de recursos y la dinamización de las bibliotecas, coordinación con servicios externos, responsabilidad en el transporte escolary en programas de habilidades sociales, prevención y resolución de conflictos y convivencia. Tareas todas ellas que ayudan a comprender la magnitud del fenómeno educador en esta comunidad, en tanto este contexto ha supuesto un claro referente en la inclusión del educador en el sistema escolar. Sin embargo, este proceso de incorporación se realizó sin una clara delimitación profesional, de ahí la disparidad en las funciones a desempeñar, algunas de las cuales han sido varias veces cuestionadas (Castillo, 2012; Castillo y Galán, 2008; González, Olmos y Serrate, 2012) por no corresponder propiamente con el perfil del educador.

\subsection{Valoración de las funciones, de acuerdo a la titulación de origen}

En un segundo momento, se realizó una comparación para verificar si existían diferencias en función de la titulación respecto a la valoración que los participantes de ambas Comunidades Autónomas realizaban de las tareas que desempeñan en su ejercicio diario. Para ello adoptamos como procedimiento la prueba de $\mathrm{T}$ para muestras independientes. En primer lugar consideramos las funciones en frecuencia de ejecución en cada comunidad y, en segundo lugar, en importancia concedida.

3. Recurso interno porque se encuentra inserto en el propio centro escolar, recogido de esta forma en el Modelo de Convivencia Escolar en la Comunidad de Castilla La Mancha (2006) y Decreto 3/2008, de la Convivencia Escolar de Castilla La Mancha. 
TABla 3. COMPARACIÓN DE MEDIAS: FUNCIONES REALIZADAS -FRECUENCIA- EN BASE A LA TITULACIÓN

\begin{tabular}{|c|c|c|c|c|c|c|c|c|c|c|c|c|}
\hline \multirow{3}{*}{ Funciones (en frecuencia) } & \multicolumn{4}{|c|}{ Castilla La Mancha $(n=27)$} & \multicolumn{2}{|c|}{$\mathrm{t}$} & \multicolumn{4}{|c|}{ Extremadura $(n=81)$} & \multicolumn{2}{|c|}{$\mathrm{t}$} \\
\hline & \multicolumn{2}{|c|}{$\begin{array}{c}\text { Educación } \\
\text { Social }(n=17)\end{array}$} & \multicolumn{2}{|c|}{ Otra $(n=10)$} & \multirow{2}{*}{$\mathrm{t}$} & \multirow{2}{*}{$\mathrm{p}$} & \multicolumn{2}{|c|}{\begin{tabular}{|c|} 
Educación \\
Social $(n=62)$
\end{tabular}} & \multicolumn{2}{|c|}{ Otra $(n=19)$} & & \multirow{2}{*}{$\mathrm{p}$} \\
\hline & $\bar{x}$ & $\mathrm{~S}_{\mathrm{x}}$ & $\bar{x}$ & $\mathrm{~s}_{\mathrm{x}}$ & & & $\bar{x}$ & $\mathrm{~S}_{\mathrm{x}}$ & $\bar{x}$ & $\mathrm{~S}_{\mathrm{x}}$ & & \\
\hline $\begin{array}{l}\text { 1. Detección y prevención } \\
\text { de factores de riesgo }\end{array}$ & 3.58 & 0.515 & 3.25 & 0.500 & 1.128 & 0.278 & 3.50 & 0.546 & 3.42 & 0.669 & 0.452 & 0.653 \\
\hline 2. Mediación en conflictos & 3.44 & 0.629 & 2.75 & 0.957 & 1.770 & 0.094 & 3.25 & 0.795 & 3.53 & 0.640 & -1.279 & 0.205 \\
\hline $\begin{array}{l}\text { 3. Organización y } \\
\text { gestión de servicios }\end{array}$ & 2.20 & 0.775 & 1.50 & 0.577 & 1.673 & 0.113 & 2.50 & 0.813 & 2.44 & 0.629 & 0.285 & 0.776 \\
\hline $\begin{array}{l}\text { 4. Programas de } \\
\text { intervención intercultural }\end{array}$ & 2.06 & 0.680 & 1.75 & 0.957 & 0.762 & 0.456 & 2.26 & 0.745 & 2.60 & 1.056 & -1.185 & 0.252 \\
\hline 5. Programas de acogida & 2.50 & 0.816 & 2.75 & 0.500 & -0.579 & 0.570 & 2.53 & 0.824 & 2.93 & 0.997 & -1.563 & 0.122 \\
\hline $\begin{array}{l}\text { 6. Programas atención } \\
\text { a la diversidad }\end{array}$ & 2.44 & 0.964 & 3.50 & 1.000 & -1.959 & 0.066 & 2.95 & 0.902 & 2.21 & 1.122 & 2.630 & 0.010 \\
\hline $\begin{array}{l}\text { 7. Programas de } \\
\text { cooperación educativa }\end{array}$ & 2.36 & 1.008 & 2.75 & 0.957 & -0.694 & 0.498 & 2.05 & 0.910 & 2.20 & 0.941 & -0.567 & 0.572 \\
\hline $\begin{array}{l}\text { 8. Programas de comunicación } \\
\text { interpersonal }\end{array}$ & 3.31 & 0.793 & 3.00 & 0.816 & 0.701 & 0.492 & 3.52 & 0.535 & 3.31 & 0.704 & 1.268 & 0.209 \\
\hline 9. Programas de participación & 2.07 & 0.997 & 2.00 & 0.000 & 0.268 & 0.793 & 2.27 & 0.887 & 2.53 & 0.516 & -1.095 & 0.277 \\
\hline 10. Escuela de padres, etc. & 2.88 & 1.310 & 1.50 & 1.000 & 1.946 & 0.067 & 2.97 & 0.868 & 2.94 & 0.574 & 0.167 & 0.868 \\
\hline 11. Programas de orientación & 2.19 & 0.981 & 2.50 & 1.000 & -0.568 & 0.577 & 2.58 & 0.944 & 2.50 & 0.855 & 0.302 & 0.763 \\
\hline $\begin{array}{l}\text { 12. Programas contra } \\
\text { absentismo escolar }\end{array}$ & 3.88 & 0.342 & 2.20 & 1.095 & 3.368 & 0.026 & 3.85 & 0.399 & 3.94 & 0.250 & -0.788 & 0.433 \\
\hline $\begin{array}{l}\text { 13. Organización de } \\
\text { actividades extraescolares }\end{array}$ & 1.94 & 0.929 & 2.00 & 1.414 & -0.109 & 0.914 & 2.64 & 0.866 & 2.57 & 0.852 & 0.283 & 0.778 \\
\hline $\begin{array}{l}\text { 14. Participar en } \\
\text { habilidades sociales }\end{array}$ & 3.38 & 0.885 & 3.00 & 0.816 & 0.768 & 0.453 & 3.61 & 0.585 & 3.75 & 0.447 & -1.066 & 0.295 \\
\hline 15. Actividad docente & 1.00 & 0.000 & 2.00 & 1.732 & -1.000 & 0.423 & 1.75 & 1.149 & 2.33 & 1.414 & -1.319 & 0.193 \\
\hline $\begin{array}{l}\text { 16. Función tutorial } \\
\text { en grupo docente }\end{array}$ & 1.54 & 0.877 & 2.50 & 1.732 & -1.069 & 0.353 & 1.88 & 1.044 & 2.33 & 1.231 & -1.312 & 0.195 \\
\hline 17. Función tutorial profesional & 1.00 & 0.000 & 1.00 & 0.000 & - & - & 1.33 & 0.730 & 1.45 & 0.934 & -0.490 & 0.626 \\
\hline
\end{tabular}


En la Comunidad Autónoma de Castilla La Mancha, el estadístico t toma el valor 3,368 (ítem 12), y tiene asociado un nivel crítico bilateral menor que 0,05 $(\mathrm{p}=0,026)$. Concluiremos, puesto que el nivel crítico es menor que 0,05, existen diferencias en función de la titulación, de tal forma que los educadores diplomados en Educación Social en Castilla La Mancha, obtienen una media mayor en dicha función $(\bar{x}=3,88)$ que los educadores con otra titulación $(\bar{x}=2,20)$, obteniendo con ello que los titulados en Educación Social se encargan con mayor frecuencia del diseño y ejecución de programas de prevención de situaciones de absentismo entre el alumnado.

En Extremadura, se observan diferencias estadísticamente significativas en el ítem $6(\mathrm{t}=2,630 ; \mathrm{p}=0,010)$, siendo la media más elevada en el grupo de educadores diplomados en Educación Social $(2,95)$ como puede verse en la Tabla 3, en este caso, los educadores titulados dedican más tiempo al diseño y desarrollo de programas de atención a la diversidad en comparación con sus homólogos no titulados en Educación Social.

En cuanto a la importancia concedida los resultados obtenidos son los que se recogen a continuación: 
TABLA 4. COMPARACIÓN DE MEDIAS: FUNCIONES REALIZADAS -IMPORTANCIA- EN BASE A LA TITULACIÓN

\begin{tabular}{|c|c|c|c|c|c|c|c|c|c|c|c|c|}
\hline \multirow{3}{*}{$\begin{array}{l}\text { Funciones (en } \\
\text { importancia) }\end{array}$} & \multicolumn{4}{|c|}{ Castilla La Mancha $(\mathrm{n}=27)$} & & \multicolumn{4}{|c|}{ Extremadura $(\mathrm{n}=81)$} & \multicolumn{2}{|c|}{4} \\
\hline & \multicolumn{2}{|c|}{$\begin{array}{c}\text { Educación } \\
\text { Social }(\mathrm{n}=17)\end{array}$} & \multicolumn{2}{|c|}{ Otra $(n=10)$} & \multirow[b]{2}{*}{$\mathrm{t}$} & \multirow[b]{2}{*}{$\mathrm{p}$} & \multicolumn{2}{|c|}{$\begin{array}{c}\text { Educación } \\
\text { Social }(n=62)\end{array}$} & \multicolumn{2}{|c|}{ Otra $(n=19)$} & \multirow[t]{2}{*}{. } & \multirow[t]{2}{*}{$\mathrm{p}$} \\
\hline & $\bar{x}$ & $s_{x}$ & $\bar{x}$ & $\mathrm{~s}_{\mathrm{x}}$ & & & $\bar{x}$ & $s_{x}$ & $\bar{x}$ & $s_{x}$ & & \\
\hline $\begin{array}{l}\text { 1. Detección y } \\
\text { prevención de factores } \\
\text { de riesgo... }\end{array}$ & 3.92 & 0.289 & 3.75 & 0.500 & 0.837 & 0.417 & 3.84 & 0.373 & 3.83 & 0.389 & 0.028 & 0.978 \\
\hline 2. Mediación en conflictos & 3.75 & 577 & 3.00 & 816 & 2.151 & 0.045 & 3.80 & 440 & 3.79 & .426 & 0.135 & 0.893 \\
\hline $\begin{array}{l}\text { 3. Organ } \\
\text { de servic }\end{array}$ & 2.81 & 0.544 & 2.75 & 0.957 & 0.177 & 0.862 & 2.87 & 0.839 & 3.14 & .770 & 1.110 & 0.270 \\
\hline $\begin{array}{l}\text { s de } \\
\text { intercultural }\end{array}$ & 3.19 & 0.544 & 3.00 & 0.000 & 0.583 & 0.567 & 2.98 & 0.707 & 3.21 & 0.893 & -1.049 & 0.297 \\
\hline 5. Progr & 3.50 & 0.632 & 3.25 & 0.500 & 0.730 & 0.475 & 3.25 & 0.671 & 3.69 & 0.630 & -2.164 & 0.034 \\
\hline $\begin{array}{l}\text { 6. Prog } \\
\text { la dive }\end{array}$ & 3.19 & 0.911 & 3.50 & 1.000 & .604 & 0.554 & 3.40 & 0.735 & 3.08 & 862 & 13 & 0.162 \\
\hline $\begin{array}{l}\text { de } \\
\text { educativa }\end{array}$ & 3.00 & 1.109 & 3.25 & 0.500 & -0.431 & 0.672 & 2.80 & 0.703 & 2.92 & 0.954 & -0.522 & 0.603 \\
\hline de & 3.69 & 0.704 & 3.50 & 0.577 & 0.490 & 0 & 3.81 & 6 & 3.80 & 0.414 & 0.083 & 0.934 \\
\hline $\begin{array}{l}\text { 9. Progr: } \\
\text { participa }\end{array}$ & 3.00 & 0.845 & 3.00 & 0.000 & 0.000 & 1.000 & 2.82 & 0.873 & 3.00 & 0.816 & -0.694 & 0.490 \\
\hline 10. Escuela de padi & 3.56 & 0.814 & 3.50 & 0.577 & 0.143 & 0.888 & 3.65 & 0.515 & 3.80 & .414 & -1.235 & 0.228 \\
\hline $\begin{array}{l}\text { 11. Pro } \\
\text { orienta }\end{array}$ & 2.67 & 1.113 & 3.25 & 1.500 & -0.871 & 0.396 & 3.06 & 0.956 & 2.86 & 0.949 & 0.734 & 0.465 \\
\hline $\begin{array}{l}\text { 12. Programas contra } \\
\text { absentismo escolar }\end{array}$ & 3.88 & 0.342 & 3.40 & 0.894 & 1.161 & 0.305 & 3.81 & 0.396 & 4.00 & 0.000 & -.319 & 0.000 \\
\hline $\begin{array}{l}\text { 13. Ory } \\
\text { activid }\end{array}$ & 2.50 & 1.211 & 2.25 & 0.957 & 0.381 & 0.707 & 2.98 & 0.764 & 2.93 & 0.616 & 0.251 & 0.802 \\
\hline $\begin{array}{l}\text { 14. Participar en } \\
\text { habilidades sociales }\end{array}$ & 3.44 & 0.9 & 3.50 & 0.577 & -0.123 & 0.904 & 3.72 & 0.45 & 4.00 & 0.000 & -3.687 & 0.000 \\
\hline 15. Actividad docent & 1.40 & 0.966 & 2.50 & 2.121 & -1.250 & 0.240 & 2.39 & 1.379 & 2.86 & 1.464 & -0.815 & 0.420 \\
\hline $\begin{array}{l}\text { 16. Función tutorial en } \\
\text { grupo docente }\end{array}$ & 2.08 & 1.240 & 2.50 & 1.732 & -0.530 & 0.604 & 2.38 & 1.134 & 2.90 & 1.197 & -1.305 & 0.198 \\
\hline $\begin{array}{l}\text { 17. Función tutorial } \\
\text { profesional }\end{array}$ & 1.27 & 0.905 & 2.00 & 1.414 & -0.983 & 0.347 & 1.64 & 0.903 & 2.00 & 1.054 & -1.085 & 0.284 \\
\hline
\end{tabular}


M. GONZÁLEZ SÁNCHEZ, S. OLMOS MIGUELÁÑEZ Y S. SERRATE GONZÁLEZ

En cuanto al grado de importancia otorgado a las funciones planteadas, en Castilla La Mancha se advierten únicamente diferencias en el ítem $2(\mathrm{t}=2,151 ; \mathrm{p}=$ 0,045), siendo la media en el grupo de educación social más elevada $(3,75)$, entendiendo por lo tanto que los profesionales titulados en Educación Social otorgan mayor importancia a las tareas de mediación en conflictos que el resto de educadores. En Extremadura, se observan diferencias en tres de los ítems propuestos, en el ítem $5(\mathrm{t}=-2,164 ; \mathrm{p}=0,034)$, que hace alusión al diseño y desarrollo de programas de acogida, en el ítem $12(\mathrm{t}=-3,819 ; \mathrm{p}=0,000)$ relativo a los programas contra absentismo escolar y en el ítem $14(\mathrm{t}=-3,687 ; \mathrm{p}=0,000)$ que se refiere a los programas dirigidos a la participación en habilidades sociales. En este caso, la media en importancia es más alta en todos los ítems en el grupo de aquellos profesionales que tienen una titulación diferente a la de educador social como puede observarse en la Tabla 4.

Esta circunstancia puede ser explicada de modo que los educadores que ejercen en la Comunidad de Extremadura, que no están titulados en Educación Social, carezcan, por un lado, de los conocimientos y habilidades necesarias y, por otro lado, del tiempo que requiere la atención a situaciones específicas que se desprenden del desempeño de funciones encaminadas a la acogida del nuevo alumnado al centro escolar, la problemática del absentismo escolar y el diseño de estrategias para trabajar las habilidades sociales con los miembros de la comunidad escolar. Tanto los educadores sociales titulados, como los educadores sin titular, otorgan mayor importancia a estas funciones referidas anteriormente en comparación a la frecuencia en que las desarrollan, sin embargo, es importante resaltar que los profesionales de la educación social tienen por formación y definición los cometidos profesionales señalados anteriormente, por lo que tiene sentido considerar que el perfil que mejor se adecúa para realizar un trabajo socioeducativo en el espacio escolar sean aquellos educadores titulados, puesto que con sus acciones, como se ha puesto de manifiesto, favorecen el proceso educativo de los alumnos y sus familias.

\section{DisCUSIÓN Y CONCLUSIONES}

Partimos de la identificación de las funciones realizadas por el educador social en los centros de enseñanza, para argumentar la existencia de un perfil profesional con los conocimientos, habilidades, destrezas y actitudes que le hacen competente para actuar y complementar a otros profesionales ante las nuevas demandas que se plantean a la educación en el contexto de la escuela actual.

Se ha analizado la situación del profesional de la Educación Social en los centros educativos de dos Comunidades Autónomas y los resultados obtenidos nos permiten señalar que el educador social está integrado en la plantilla de los centros educativos, que se encuentra desempeñando tareas encaminadas a dar respuesta a aquellas necesidades surgidas en el entorno escolar y para las que no ha existido, hasta el momento, un profesional específico. 
Nos encontramos ante un agente educativo cuya función, al menos aparentemente, no es impartir docencia, y sí dedicarse a realizar todas aquellas tareas que no encajan en lo docente. Es un profesional que trabaja de forma directa con la comunidad educativa favoreciendo los procesos de enseñanza y mejorando el clima escolar.

Los sectores de la comunidad escolar a los que principalmente se dirige la intervención de los educadores son los alumnos y sus familias, no quedando reflejada su interacción con otros agentes educativos externos a los centros e instancias sociales, actividad que debería ser abordada con la finalidad de poner de manifiesto el rol mediador del educador social y su figura nexo de unión del centro con el contexto/entorno más cercano.

Se ha apreciado una fuerte coincidencia entre las intervenciones más frecuentes (ante factores de riesgo, en el desarrollo de mecanismos que fomenten la convivencia, en programas de comunicación interpersonal y el desarrollo de habilidades sociales) y la importancia que el educador les atribuye. Por su parte, se ha encontrado mayor disparidad en las funciones consideradas más importantes (intervención intercultural, diseño de programas de acogida o desarrollo de escuelas de padres) respecto a la frecuencia de ejecución, fundamentalmente entre aquellos educadores no titulados. Todas estas funciones vienen a coincidir con tareas propias del perfil del educador social en otros espacios y contextos (Vallés, 2011) corroborando, tal y como se pone de manifiesto en otros estudios (Conde, 2012; Sarrado y Fernández-Díaz, 2012), que su desarrollo favorece el proceso el aprendizaje y desarrollo social de los sujetos.

Su quehacer en el instituto de las comunidades examinadas se concentra fundamentalmente en torno a tareas como la detección y prevención de factores de riesgo, mediación en conflictos de convivencia y su resolución, diseño y desarrollo de programas de comunicación interpersonal, desarrollo de programas contra el absentismo escolar y participación en el desarrollo de habilidades sociales. El educador social escolar, con el desarrollo de este conjunto heterogéneo de funciones, ofrece respuestas integrales a través de la realización de un trabajo exhaustivo encaminado principal y fundamentalmente al desarrollo de la competencia social del alumnado con el que trabaja.

Diversidad de funciones y tareas agrupadas como plantean Ortega y Mohedano (2012) en un modelo específico y especializado de actuación del educador social, cuyos profesionales provienen mayoritariamente de la titulación de Educación Social y que se centra en acciones de prevención, tratamiento y control ante situaciones ya descritas como el absentismo o la violencia escolar, o como el fomento de aspectos esenciales como la convivencia o las habilidades sociales. En estos espacios comunitarios, no obstante, queda aún camino por recorrer en cuanto a la ubicación formal, en tanto hasta el momento no pertenecen a las plantillas académicas ni docentes de los centros escolares, por lo que no poseen los mismos derechos y obligaciones que sus compañeros docentes, profesores y profesionales específicos. 
M. GONZÁLEZ SÁNCHEZ, S. OLMOS MIGUELÁÑEZ Y S. SERRATE GONZÁLEZ

A partir de los resultados obtenidos en investigaciones previas (Ortega et al., 2010) se extrae que el modelo especializado de actuación del educador social coexiste con un modelo más general, docente y de apoyo en aquellas comunidades en las que aun no existiendo plazas específicas para el educador social, interviene en el sistema escolar a partir de puestos de profesor de servicios a la comunidad o profesor de ciclos formativos, es el caso de la Comunidad Autónoma de Castilla y León. En estos casos, el profesional tiene asignadas funciones docentes y de asesoramiento dentro de los Departamentos o Equipos de Orientación.

En la actualidad tanto uno como otro constituyen modelos incompletos y reduccionistas de las posibilidades que el educador social puede aportar y ofrecer al marco escolar. La tendencia debe ser ampliar la mirada desde la que se define el perfil del educador social escolar y dirigirla hacia un modelo de acción socioeducativa, donde el educador social se centre, principalmente, en tareas de enseñanza centradas en contenidos sociales (Meerovich y Pérez, 2013), que además interviene y colabora con el resto de personal docente en tareas dirigidas a ofrecer una educación integral, trabajar para mantener la convivencia pacífica y solventar dificultades y problemas que surjan en el quehacer diario de los centros de enseñanza a partir de prácticas concretas de educación social que otros profesionales no pueden desempeñar.

Asumiendo que cualquier tipo de clasificación o decálogo de funciones tiene sus riesgos e imprecisiones, presentamos un elenco de funciones a desempeñar por el educador social en distintos espacios escolares (ver Tabla 5), que se desprenden de la investigación realizada y tomando como apoyo las recopilaciones de funciones realizadas por otros autores como Méndez (2007), conscientes de que al ser dinámicas deben ser constantemente supervisadas, evaluadas y reformuladas para adaptarse a las necesidades de cada momento y siempre adecuadas a las necesidades de los centros. 
M. GONZÁLEZ SÁNCHEZ, S. OLMOS MIGUELÁÑEZ Y S. SERRATE GONZÁLEZ

PENSAMIENTO Y ACCIÓN SOCIOEDUCATIVA EN CONTEXTOS DE ENSEÑANZA SECUNDARIA...

TABLA 5. FUnCIONES DEL EDUCADOR SOCIAL ESCOLAR (ELABORACIÓN PROPIA)

\begin{tabular}{|c|c|}
\hline \multirow{2}{*}{$\begin{array}{l}\text { DESEMPEÑO } \\
\text { CON FAMILIAS }\end{array}$} & Intervención en/con familias con diferentes problemáticas \\
\hline & Colaboración con las asociaciones de madres y padres de alumnos \\
\hline \multirow{5}{*}{$\begin{array}{l}\text { DESEMPEÑO } \\
\text { CON ALUMNADO }\end{array}$} & Prevención del absentismo escolar \\
\hline & Detección y prevención de factores de riesgo \\
\hline & Mediación en conflictos de convivencia y su resolución \\
\hline & $\begin{array}{l}\text { Realización de talleres y campañas sobre salud sexual y educación } \\
\text { afectivo-sexual }\end{array}$ \\
\hline & $\begin{array}{l}\text { Seguimiento socioeducativo del alumnado en riesgo o con } \\
\text { problemas }\end{array}$ \\
\hline \multirow{3}{*}{$\begin{array}{l}\text { DESEMPEÑO CON } \\
\text { PROFESIONALES }\end{array}$} & Colaboración con la acción tutorial \\
\hline & Colaboración en la realización de evaluaciones psicopedagógicas \\
\hline & $\begin{array}{l}\text { Asesoramiento respecto a la intervención con alumnado de manera } \\
\text { individualizada }\end{array}$ \\
\hline \multirow{2}{*}{$\begin{array}{l}\text { DESEMPEÑO } \\
\text { CON EL ENTORNO }\end{array}$} & $\begin{array}{l}\text { Coordinación con Servicios Sociales sobre familias y alumnos más } \\
\text { desfavorecidos para su seguimiento }\end{array}$ \\
\hline & $\begin{array}{l}\text { Generación de redes sociales, contextos, procesos y recursos } \\
\text { educativos y sociales }\end{array}$ \\
\hline \multirow{5}{*}{$\begin{array}{l}\text { DESEMPEÑO } \\
\text { EN EL CENTRO }\end{array}$} & $\begin{array}{l}\text { Gestión y coordinación de las aulas de convivencia y desarrollo de } \\
\text { programas de mejora de la convivencia en los centros }\end{array}$ \\
\hline & $\begin{array}{l}\text { Diseño y desarrollo de programas de habilidades sociales, } \\
\text { comunicación interpersonal, educación en valores, prevención de } \\
\text { drogodependencias... }\end{array}$ \\
\hline & $\begin{array}{l}\text { Responsable de ludotecas, programas de animación a la lectura y } \\
\text { dinamización de recreos }\end{array}$ \\
\hline & Realización de actividades de acogida \\
\hline & $\begin{array}{l}\text { Información y asesoramiento en la información sobre becas y ayudas } \\
\text { al estudio }\end{array}$ \\
\hline
\end{tabular}

En una sociedad que demanda de la educación no sólo formación sino respuesta a otro tipo de problemas, el perfil del educador social debe responder a esta demanda, lo que justifica la incorporación y el reconocimiento de este profesional en los centros donde exista riesgo o conflictividad social, y en aquellos que sin ningún tipo de problemática social trabajen en clave comunitaria y preventiva.

Convenimos en optar por la colaboración interdisciplinar que señala Cabrera (2004), en cualquier caso, la intervención global del educador ha de contemplarse y debe irse construyendo y consolidando desde la colaboración interprofesional con los profesionales de los centros. Intervención global y colaborativa como es la desarrollada por García y Blázquez (2006) al manifestar que la incorporación de educadores en los centros escolares debe ofrecer nuevas posibilidades al sistema.

Hablamos de un trabajo fundamentado en la colaboración con el resto de agentes educativos, que persiga el desarrollo social y personal del alumnado, 
M. GONZÁLEZ SÁNCHEZ, S. OLMOS MIGUELÁÑEZ Y S. SERRATE GONZÁLEZ

PENSAMIENTO Y ACCIÓN SOCIOEDUCATIVA EN CONTEXTOS DE ENSEÑANZA SECUNDARIA...

englobado el ejercicio profesional del educador social en el contexto amplio del centro escolar y enmarcando el desempeño de sus acciones bajo objetivos comunitarios y de integración de los sujetos con los que se trabaja en el contexto macrosocial, a través del desarrollo de planes y proyectos trascendentales que fomenten el bienestar y ofrezcan respuestas a las situaciones conflictivas o de necesidad que se manifiestan en el microcontexto escolar.

\section{REFERENCIAS BIBLIOGRÁFICAS}

Arnal, J.; Del Rincón, D. y Latorre, A. (1992) Investigación educativa. Fundamentos y metodología. Barcelona, Labor.

BARRANCO, R.; DíAz, M. y Fernández, E. (2012) El Educador social en la educación secundaria. Valencia, Nau Libres.

Bautista-Cerro, M. J. y Melendro, M. (2011) Competencias para la intervención socioeducativa con jóvenes en dificultad social. Educación XXI, 14, 1, 179-200. doi: http://dx.doi. org/10.5944/educxx1.14.1.268.

Beltrán, J. (2007) La escuela del siglo xxi, jugar en serio, en GiRó, J. (ed.) La escuela del siglo XXI: la educación en un tiempo de cambio acelerado. Logroño, Universidad de la Rioja, 1-16.

Bernete, F. (2010) Usos de las Tic, relaciones sociales y cambios en la socialización de las y los jóvenes. Revista de Estudios de Juventud, 88, 97-114.

Bertalanffy, L. V. (1976) Teoría General de los Sistemas. México, Fondo de Cultura Económica.

Bolívar, A. (2013) Liderazgo educativo. Aula de Innovación Educativa, 221, 12-17.

Cabrera, P. J. (2004) La vida al raso. Educación Social, 27, 11-20.

Calero, J.; Choi, A. y Waisgrais, S. (2010) Determinantes del riesgo de fracaso escolar en España: una aproximación a través de un análisis logístico multinivel aplicado a PISA2006. Revista de Educación, n. ${ }^{\circ}$ extraordinario, 225-256.

Castillo, M. (2008) Els recursos educatius amb alumnes disruptius a l'Eso: mirades i reflexions. Perspectiva Escolar, 325, 60-66.

Castillo, M. (2012) La intervención del educador social en el marco educativo formal. Barcelona, UOC.

Castillo, M. (2013) La aportación de los educadores y educadoras sociales a la escuela: nuevas competencias, nuevas posibilidades. Revista de Educación Social, 16, 1-11.

Castillo, M. y Galán, D. (2008) El papel de los educadores sociales en los centros de secundaria: una propuesta para el debate. Educación Social. Revista de Intervención Socioeducativa, 38, 121-133.

Coll, C. y MarTín, E. (2009) Curriculum reforms and the field of curriculm in Spain, en PINAR, W. F. (ed.) International Handbook of Curriculum Research ( $2^{\text {nd }}$ edition). Mahwah, New Jersey, Lawrence Earlbaum Associates.

CONDE, S. (2012) Estudio sobre la gestión de la convivencia escolar en centros de Educación Secundaria de Andalucía: una propuesta de evaluación basada en el Modelo EFQM. Tesis Doctoral. Universidad de Huelva.

Córdoba, F.; Del Rey, R. y Ortega, R. (2010) Abordando la conflictividad social en el instituto de secundaria, en ImBERnón, F. (coord.) Procesos y contextos educativos: Enseñar en las instituciones de Educación Secundaria. Barcelona, Graó, 111-130. 
CSFP (2013) Abandono escolar temprano. Factores de abandono y factores de continuidad. Intervenciones posibles desde los centros educativos. Centro Superior de Formación del Profesorado, Junta de Castilla y León.

DE LEÓn, B. (2011) La relación familia-escuela y su repercusión en la autonomía y responsabilidad de los niños/as. XII Congreso Internacional de Teoría de la Educación. Barcelona, 20-22 de octubre.

Estrada, O. (2012) El profesor ante la formación de valores. Aspectos teóricos y prácticos. Teoría de la Educación. Educación y Cultura en la Sociedad de la Información, 13, 3, 240-267.

Fernández, M.; Mena, L. y Riviere, J. (2010) Fracaso y abandono escolar en España. Barcelona, Fundación "la Caixa».

Garaigordobil, M. (2011) Prevalencia y consecuencias del Cyberbullying: una revisión. International Journal of Psychology and Psychological Therapy, 11, 2, 233-254.

García, J. y BlázQuez, A. (2006) El educador social en la educación secundaria. Educación Social, 32, 39-58.

Gobernado, R. (2007) El cuidado de los hijos y el género. Informe FIPROS. Madrid, Ministerio de Trabajo y Asuntos Sociales.

González, M.; Olmos, S. y Serrate, S. (2012) Los centros educativos, nuevo ámbito emergente de intervención de los educadores sociales, en NieTo, E.; Callejas, A. I. y Jerez, O. (eds.) Las competencias básicas. Las competencias profesionales del docente. Ciudad Real, Ediciones Universidad de Castilla La Mancha, 523-534.

GonZÁlez, M. y SERRATE, S. (2013) El educador social en la resolución de problemas y mejora de la convivencia en el contexto de los centros escolares, en Pérez, M. C. y Molero, M. M. (eds.) Variables psicológicas y educativas para la intervención en el ámbito escolar. Almería, Asociación Universitaria de Educación y Psicología, 54-62.

Guerrero, E. y RuBio, J. C. (2008) Fuentes de estrés y Síndrome de "Burnout» en orientadores de institutos de Enseñanza Secundaria. Revista de Educación, 347, 229-254.

Hargreaves, A. y Fink, D. (2006) Estrategias de cambio y mejora en educación caracterizadas por su relevancia, difusión y continuidad en el tiempo. Revista de Educación, 339, 43-58.

Kerlinger, F. y Lee, H. (2002) Investigación del Comportamiento, Métodos de Investigación en Ciencias Sociales. México, McGrawHill.

Krenz, A.; Casas, C.; Aguilar, O. y Carbó, M. J. (2009) La convivencia escolar desde el Enfoque Sistémico. El niño en la encrucijada entre sociedad, familia y escuela. Información Psicológica, 95, 46-61.

Losada, L.; MuÑoz, J. M. y Espiñeira, E. M. (2015) Perfil, funciones y competencias del educador social a debate: análisis de la trayectoria de la formación de profesionales de la educación social. Educación Social. Revista d'Intervenció Socioeducativa, 60, 59-76.

Martínez, C.; Krichesky, G. J. y García, A. (2011) El orientador escolar como agente interno de cambio. Revista Iberoamericana de Educación, 54, 107-122.

MarTínez, R. y De ANDRÉs, E. (2011) Informe sobre el impacto de la conciliación de la vida familiar y laboral sobre el bienestar de la infancia. Madrid, EFR Observatorio.

Matamales, R. (2007) Del absentismo escolar al ausentismo social, en Giró, J. (ed.) La escuela del siglo XXI: la educación en un tiempo de cambio social acelerado. Logroño, Universidad de la Rioja, 1-7.

Mazorra, P. (2008) El conflicto en la escuela: una oportunidad de aprendizaje. Bordón, 60 (4), 125-136. 
M. GONZÁLEZ SÁNCHEZ, S. OLMOS MIGUELÁÑEZ Y S. SERRATE GONZÁLEZ

PENSAMIENTO Y ACCIÓN SOCIOEDUCATIVA EN CONTEXTOS DE ENSEÑANZA SECUNDARIA...

Meerovich, M. y Pérez, G. (2013) Educación social y educación media. Nuestra posición de enseñantes en torno a la ampliación de la oferta y las miradas. Revista de Educación Social, 16, 1-18.

MÉNDEZ, M. (2007) La universidad ante el reto de simular la educación social en la escuela. V Congreso estatal de las educadoras y educadores sociales. Toledo, 27-29 de septiembre.

Morales, P.; Urosa, B. y Blanco, A. (2003) Construcción de escalas de actitudes tipo Likert. Madrid, La Muralla.

Mora-Merchán, J.; Ortega, R.; Calmaestra, J. y Smith, P. K. (2010) El uso violento de la tecnología: Cyberbullying, en Ortega, R. (ed.) Agresividad injustificada Bullying y Violencia escolar. Madrid, Alianza, 189-209.

Moreno, A. y Sú́rez, C. (2010) Las comunidades virtuales como nuevas formas de relación social: elementos para el análisis. Espéculo. Revista de Estudios Literarios, 43.

Moreno, T. (2010) La relación familia-escuela en secundaria: algunas razones del fracaso escolar. Revista de Currículum y Formación del Profesorado, 14, 2, 241-255.

OrTEgA, J. (2005) La educación a lo largo de la vida: la educación social, la educación escolar, la educación continua... Todas son educaciones formales. Revista de Educación, $338,167-175$.

Ortega, J.; González, M.; Froufe, S.; Rodríguez, M. J.; Sobrón, I.; Calvo, R. et al. (2010) Situación, praxis y demandas profesionales de intervención socioeducativa en el Sistema Escolar de Castilla y León. Universidad de Salamanca-IUCE.

Ortega, J. y Mohedano, J. (2012) Educadores Sociales Escolares, conceptos y modelos. XXV Seminario Interuniversitario de Pedagogía Social. Talavera de la Reina, 29-30 de noviembre.

Ortega, R.; Calmaestra, J. y Mora-Merchán, J. (2008) Cyberbullying. International Journal of Psychology and Psychological Therapy, 8, 2, 183-193.

Ortega-Reyes, J. I. y González-Bañales, D. L. (2015) Impacto del Cyberbullying en el rendimiento académico de estudiantes de nivel medio superior. México, Instituto Universitario Anglo Español.

PÉREz, G. (2008) Nuevos problemas, nuevos retos en educación social. Bordón, 4, 7-12.

PÉrez, R. (2010) Sentido profundo de la orientación. Revista Española de Orientación y Psicopedagogía, 21, 2, 207-219. http://dx.doi.org/10.5944/reop.vol.21.num.2.2010.11524.

Pérez de Guzmán, M. L. (2008) La mediación en los centros educativos, el educador social como mediador. Bordón, 60 (4), 79-87.

Pineda, J. A. y García, F. F. (2014) Convivencia y disciplina en el espacio escolar: discursos y realidades. Scripta Nova. Revista Electrónica de Geografía y Ciencias Sociales, 496, $05,1-21$.

Rahona, M. y Morales, S. (2013) Educación e inmigración en España: desafíos y oportunidades. Madrid, OEI.

SÁEz, J. (2005) La profesionalización de los educadores sociales: construcción de un modelo teórico para su estudio. Revista de Educación, 336, 129-139.

SÁNchez, J. (2008) Servicios a la Comunidad: un referente para la concreción del perfil socioeducativo de los educadores sociales en la escuela. Bordón, 60, 4, 41-50.

SARRAdo, J. J. y Fernández-Díaz, E. (2012) El educador social en centros educativos. Una propuesta actualizada de sus competencias profesionales en entornos complejos. Madrid, Dykinson.

Tribó, G. (2008) El nuevo perfil profesional de los profesores de secundaria. Educación XXI, 11, 183-209. 
114 M. GONZÁLEZ SÁNCHEZ, S. OLMOS MIGUELÁÑ̃ZZ Y S. SERRATE GONZÁLEZ PENSAMIENTO Y ACCIÓN SOCIOEDUCATIVA EN CONTEXTOS DE ENSEÑANZA SECUNDARIA...

Unceta, S. (2006) Cambios sociales y educación. Notas para el debate. Revista de Educación, 347, 419-432.

VALLÉs, J. (2011) Análisis y valoración de las funciones de los educadores sociales en España. (Tesis Doctoral). Universidad Nacional de Educación a Distancia. 